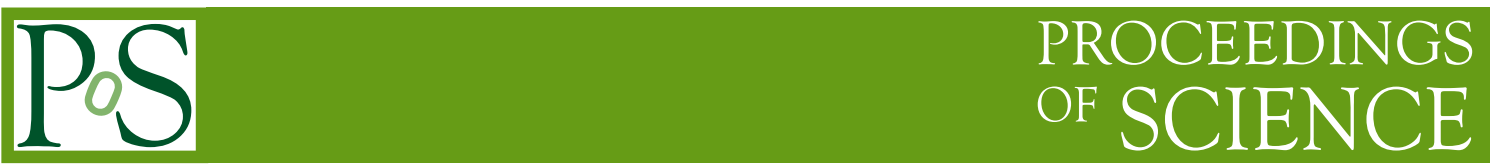

\title{
Development of new photodetectors
}

\author{
Andrea WILMS** \\ GSI, Helmholtzzentrum für Schwerionenforschung, Darmstadt, Germany \\ E-mail: A.Wilms@gsi.de
}

During the last years the experimental demands on photodetectors used in several HEP experiments have increased enormously. Aside from the financial point of view, the space requirements for many detector sub-systems lead to the usage of photosensors which have to be very compact, but providing as much granularity as possible. The device performances have to be optimized concerning the required time, spatial or energy resolutions needed for different physical applications. In addition the new generation of photodetectors has to deal with high experimental count rates and for some applications has to offer an internal signal amplification apart from the requirement of low power consumption. Due to the fact, that several detector components have to be read out during their operation inside a high magnetic field, the usage of conventional photomultiplier tubes is more or less precluded.

This paper will give a brief overview of two main developments on the sector of photodetectors, which are reaching more and more importance for high and medium energy experiments: Avalanche Photo Diodes (APDs) with large active areas and Silicon Photomultipliers (SiPMs) operating at bias voltages above breakdown.

XLVIII International Winter Meeting on Nuclear Physics in Memoriam of Ileana Iori

25-29 January 2010

Bormio, Italy

\footnotetext{
* Speaker.

${ }^{\dagger}$ Special thanks are addressed to Johann Marton and his team, providing several results of their G-APD characterization measurements shown in this paper.
} 


\section{Avalanche Photo Diodes (APDs)}

These semiconductors are mainly used in electromagnetic calorimetry, where a high energy resolution has to be achieved and the whole detector is mounted inside a magnetic field. Due to their internal structure they could reach gain vaules up to a few hundred and have very well known characteristics due to the enormous R\&D work done for the electromagnetic calorimeter readout of the CMS-ECAL. Since their application in CMS at Cern the active area size of these devices has tremendously increased from $(5 \times 5) \mathrm{mm}^{2}(\mathrm{CMS})$ to available types with $(30 \times 30) \mathrm{mm}^{2}$ active area. Nowadays large active area APDs are no longer only available as square shaped photosensors, even first rectangular types have been built and are planned e.g. to be used for the readout of the PANDA electromagnetic calorimeter, for which they are currently under investigation.

\subsection{Operation principle}

Inside a semiconductor incident light with energies larger than the bandgap energy ( $\left.E_{\text {bandgap }}\right)$ creates electron hole pairs inside the depletion layer. In case of devices made out of silicon with $E_{\text {bandgap }}=1.12 \mathrm{eV}$ light with $\lambda<1100 \mathrm{~nm}$ could be detected. Because of the high internal electric field inside an avalanche photo diode (field strenght above $10^{4} \mathrm{~V} / \mathrm{cm}$ ) the generated carriers escape the collisions with the crystal lattice which leads to an ionization of the lattice. Therefore even more electron hole pairs will be generated like in a chain reaction: this process is called the avalanche multiplication of photocurrent (begins at E-field strength value reaches $\approx\left(2 \times 10^{5}\right) \mathrm{V} / \mathrm{cm}$ ).

\subsection{QE, Gain, Dark current \& Excess Noise Factor}

Concerning the handling of APDs with such a large active area it has to be ensured that the measurement of the gain $\mathrm{M}$ of these devices is independent of the position on the surface (x,y) where the light is coupled into the device. That means gain-surface uniformity has to be guaranteed and is the pre-condition for a proper measurement of the gain e.g. depending on the applied bias voltage.

The quantum efficiency $\mathrm{QE}$ is defined as the ratio between the number of generated photo electrons inside the device and the number of incident photons. This quantity is e.g. and not only affected by the material of the passivation layer used. In principle those layers are made out of $\mathrm{SiO}_{2}$ or $\mathrm{Si}_{3} \mathrm{~N}_{4}$ with the properties shown in table 1. As shown in the table a possible reduction of

\begin{tabular}{c|c|c} 
& Bandgap energy [eV] & Refraction index n \\
$\mathrm{SiO}_{2}$ & 8.1 & 1.5 \\
\hline $\mathrm{Si}_{3} \mathrm{~N}_{4}$ & 5.9 & 1.7 \\
\hline
\end{tabular}

Table 1: Important properties of the mainly used passivation layer materials: the bandgap energy is responsible for the wavelength sensitivity and the refraction index $n$ has influence on reflection losses.

$\mathrm{QE}$ as a result of passivation layer material is mainly caused by reflection losses $\mathscr{R}$. The transmission fraction of the incident light through the passivation layer could therefore be calculated via $\mathscr{T}=1-\mathscr{R}$, with $\mathscr{R}=\left[\left(n_{2}-n_{1}\right) /\left(n_{2}+n_{1}\right)\right]^{2}$. The result of a QE measurement is shown on the 

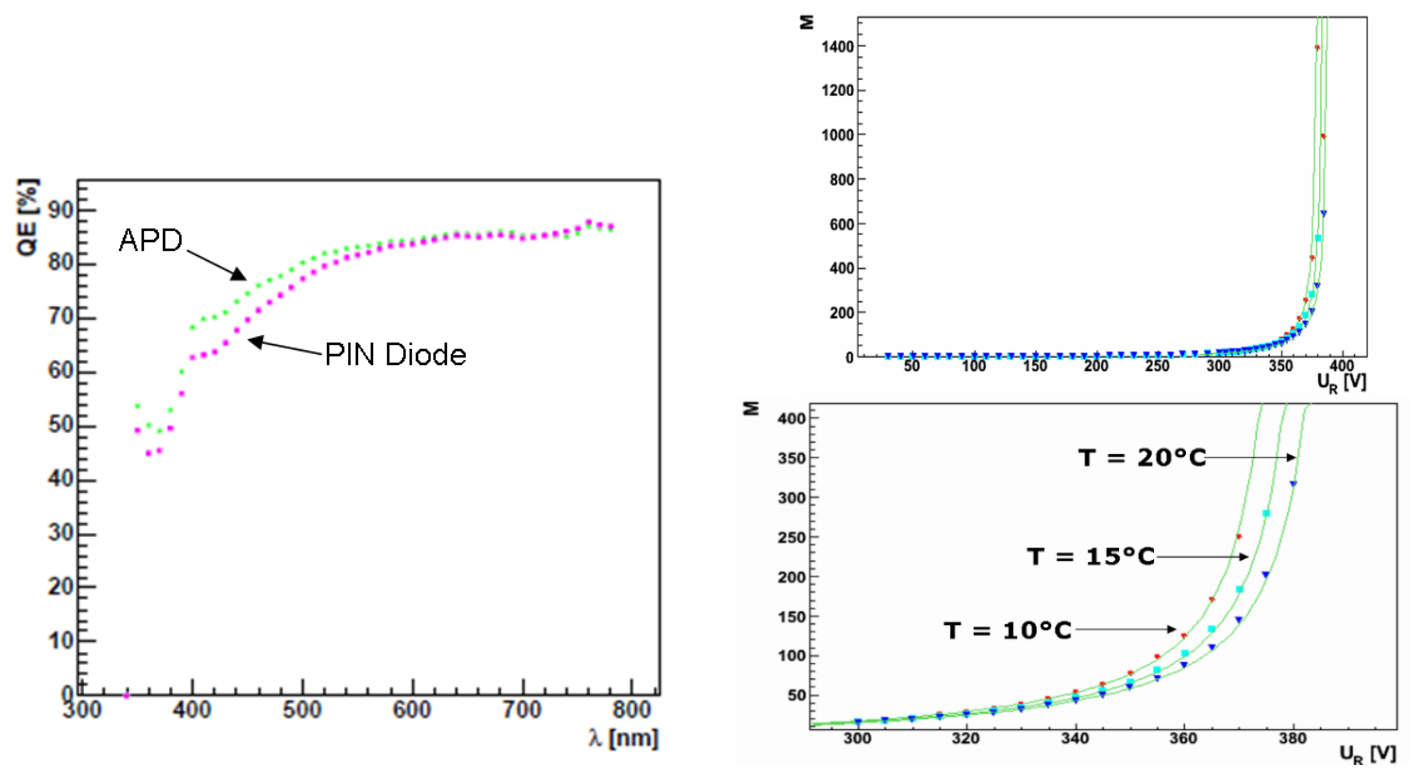

Figure 1: Left: Quantum efficiency of a large area APD compared to the QE of a PIN diode. Right: Gain measurement of one single APD at three different temperatures: $T=20^{\circ} \mathrm{C}, \mathrm{T}=15^{\circ} \mathrm{C}$ and $\mathrm{T}=10^{\circ} \mathrm{C}$. Each gain curve could be described by using a slightly modified version of the Miller-Formula [1]: $M\left(U_{R}\right)=\frac{1}{1-\left(\frac{U_{R}}{U_{B r}}\right)^{n}}$ shown in green (with $n$ : concavity index). The plot below shows the zoom of the measurement inside the important gain region of the PANDA EMC readout chain.

left side of Fig. 1 compared to the values evaluated for a typical PIN diode, the right one shows the results of such a gain measurement of one APD at three different temperatures, where the different corresponding values of the breakdown voltage $U_{B r}$ can be seen.

The internal gain $M$ of an APD depends on the applied bias voltage $\left(U_{R}\right)$ as well as on the device temperature and is determined by measuring the photo current (illumination current - dark current) of the diode at a fixed wavelength value:

$$
M\left(U_{R}\right)=\frac{I_{\text {photo }}\left(U_{R}\right)}{I_{\text {photo }}(M=1)} .
$$

The dark current $I_{d}$ of an APD consists of two different parts: the surface leakage current $I_{d s}$, which flows through the interface between the pn junction and the passivation layer, and the bulk dark current $I_{d b}$, which is an internal current generated inside the Si substrate and is multiplied by the internal gain $\mathrm{M}$ of the diode. Therefore the overall dark current of an APD at a given temperature could be written as:

$$
I_{d}=I_{d s}+M \cdot I_{d b}
$$

The temperature dependence of $I_{d}$ is clearly visible in the plots shown in Fig. 2.

The excess noise factor $F(\lambda)$ describes the fluctuations of the avalanche gain at a designated bias voltage value and has therefore influence on the noise performance of the device. The origin of this effect are random fluctuations in the distance travelled by carriers between ionizing collisions. These fluctuations end up in a rise of fluctuations in the total number of secondary generated charge 

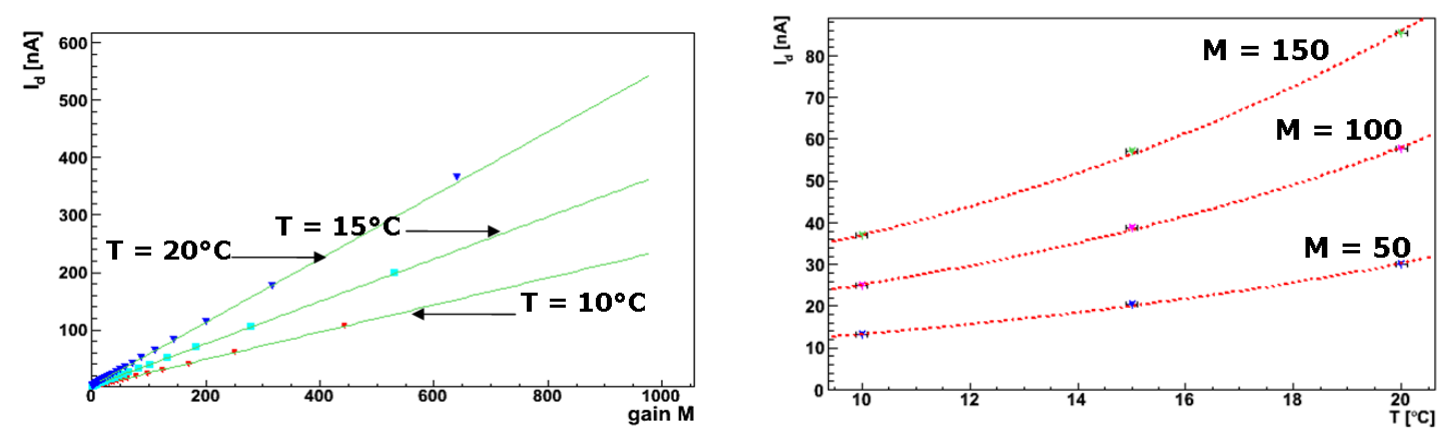

Figure 2: Left: Measured dark current $I_{d}$ of a large area APD for three different temperatures depending on the internal gain M. Right: Temperature dependence of the dark current measured for three different gain values $\mathrm{M}$.

carriers inside the diode which leads to the observed fluctuations of the measured gain value. At room temperature the excess noise factor of an APD used in PANDA for a given gain of $M=50$ is e.g. $F=1.38$.

\section{Silicon Photomultipliers (SiPMs)}

Those devices are also known as MPPCs, G-APDs or AMPDs. Due to their pixel structure they are well suitable for applications in which high spatial resolution is required. Each pixel (100 pixels up to 1000 pixels or more are available nowadays) of these devices operates in the so called 'Geiger Mode' and outputs a signal in case of photon detection. Therefore the signal output of such a SiPM is the sum of the single outputs of each pixel. Due to the internal Geiger discharge process these devices are very fast and could be used in applications in which time resolution or fast timing is desirable. In contrast to APDs they operate at bias voltages above the breakdown voltage (overvoltage) leading to the fact that extremely high gains (up to typ. $10^{6}$ ) could be reached. Therefore they are well suited for low light level detection applications up to single photon counting utilizations even though their usage as miniaturized photomultipliers for gamma detection with scintillators is quickly growing.

\subsection{Geigermode operation principle}

Photo detectors operating in Geigermode use bias voltages $U_{R}$ higher than the breakdown voltage $U_{B r}$. The parameter $U_{R}-U_{B r}=\Delta V$ is called overvoltage. Due to the high overvoltage the electric field inside the device is so high, that very huge gain values $\left(10^{5}\right.$ up to $\left.10^{6}\right)$ could be reached. That means that even very low light input creates a discharge inside the device structure (Geiger discharge).

A typical photon distribution and the corresponding pulse height spectrum of a SiPM, with a structure schematically shown in Fig. 3, are shown in Fig. 4. 

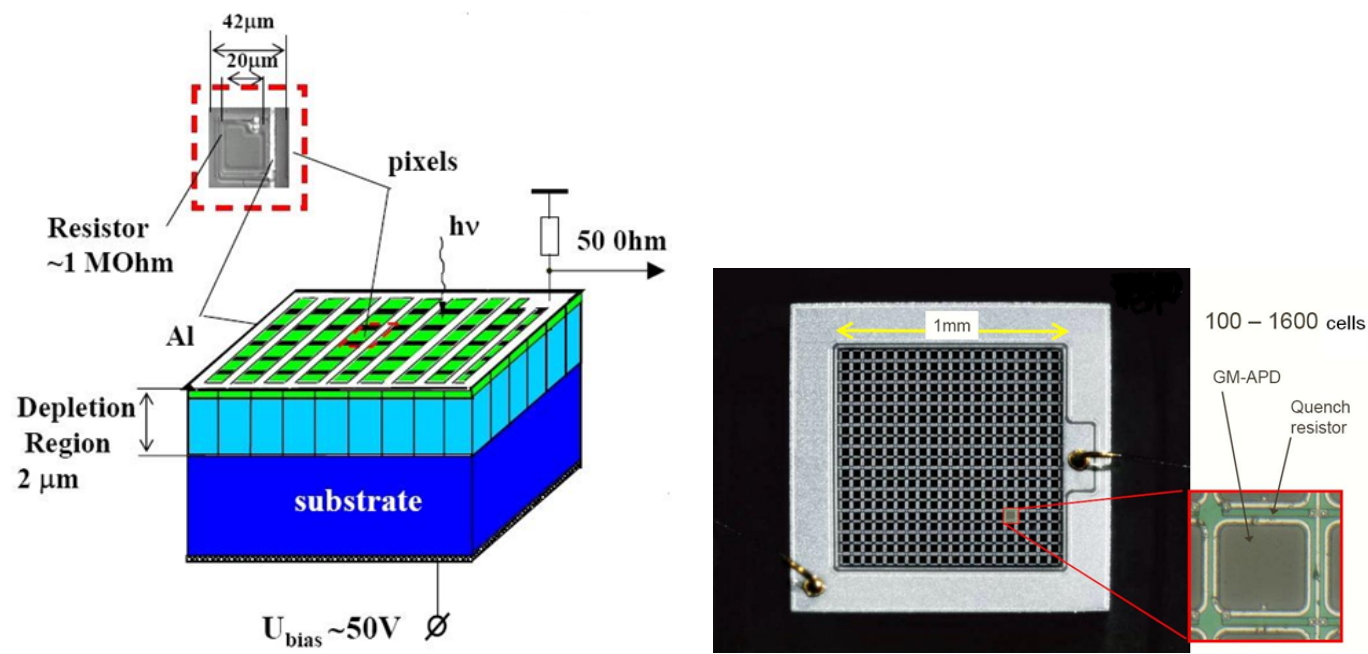

Figure 3: Left: Schematics of the structure of a SiPM including the quenching resistors connected to each pixel. Right: Photo of a typical SiPM, where the space needed for the resistors is clearly seen. Pictures taken from [8], [9].
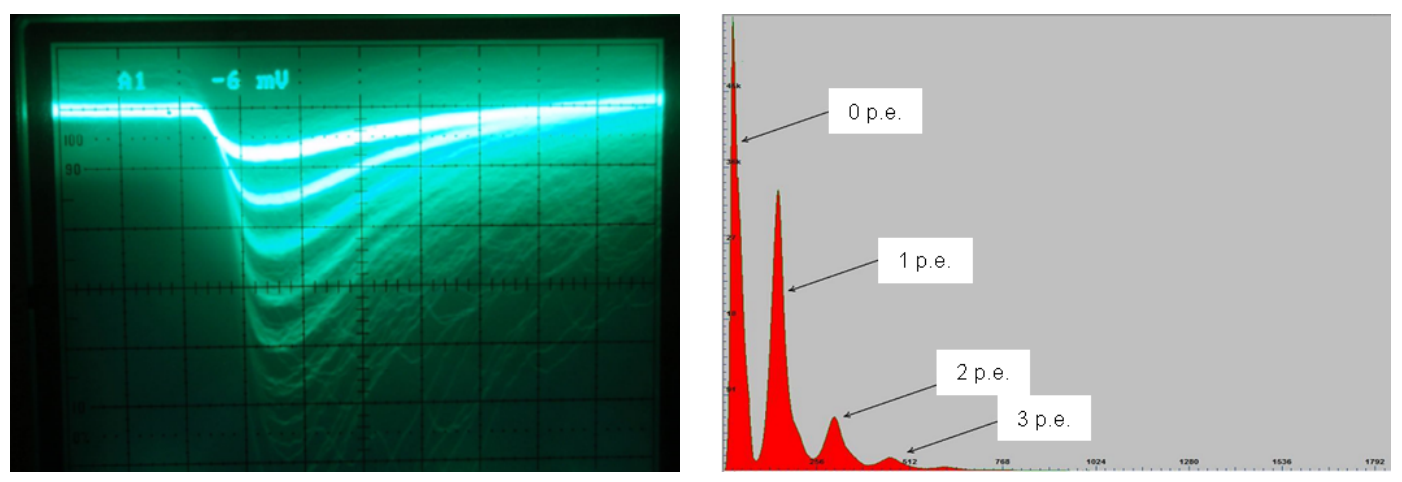

Figure 4: Left: Typical oscilloscope picture of the pulse distribution measured with a SiPM [3]. Right: The corresponding pulse height spectrum, where the signals of the incident number of photons are clearly separated from each other (taken from [3]).

\subsection{SiPM gain measurement}

The gain of a SiPM could be estimated from the measured output charge of the device at one fixed bias voltage via the equation:

$$
\operatorname{Gain}\left(U_{R}=\text { const } .\right)=\frac{\Delta q}{1.6 \cdot 10^{-19} C},
$$

where $\Delta q$ is determined as shown in Fig. 5 and has to be divided by the elementary charge. To get optimal results the parameter $\Delta q$ should be measured several times by using a certain number of peaks and the averaged value should be used for gain determination purposes. 


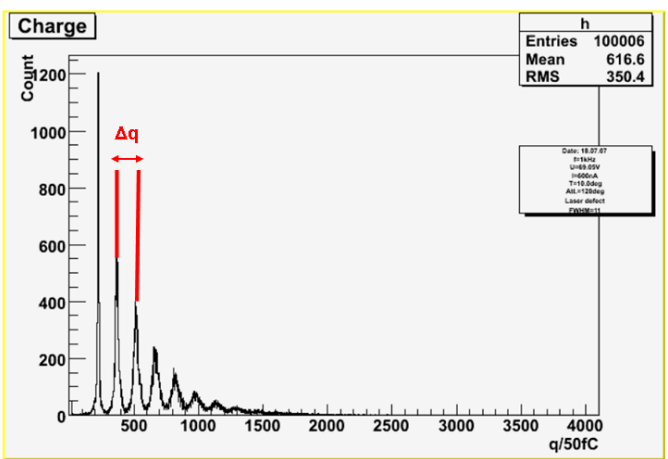

Figure 5: Measurement of the SiPM gain by determination of the parameter $\Delta q$ from the taken data.

\subsection{Photon detection efficiency (PDE)}

Compared to the QE of an APD the photon detection efficiency PDE is defined as:

$$
P D E=Q E \cdot \varepsilon \cdot p_{\text {aval }}
$$

The factor $\varepsilon$ is called 'fill factor' and describes the ratio between effective pixel size and total pixel size. That means that the fill factor has got a trade-off relation with the total pixel number, whereas the total number of pixels determines the dynamic range of the device. The influence of the fill factor on the PDE of a SiPM is exemplarily shown in Fig. 6, where also a PDE measurement taken from [4] is shown. The parameter $p_{\text {aval }}$ describes the probability of a, by an incident photon created, electron-hole pair triggering an avalanche. This avalanche probability depends on the position where the primary e-h pair is generated and on the applied overvoltage.
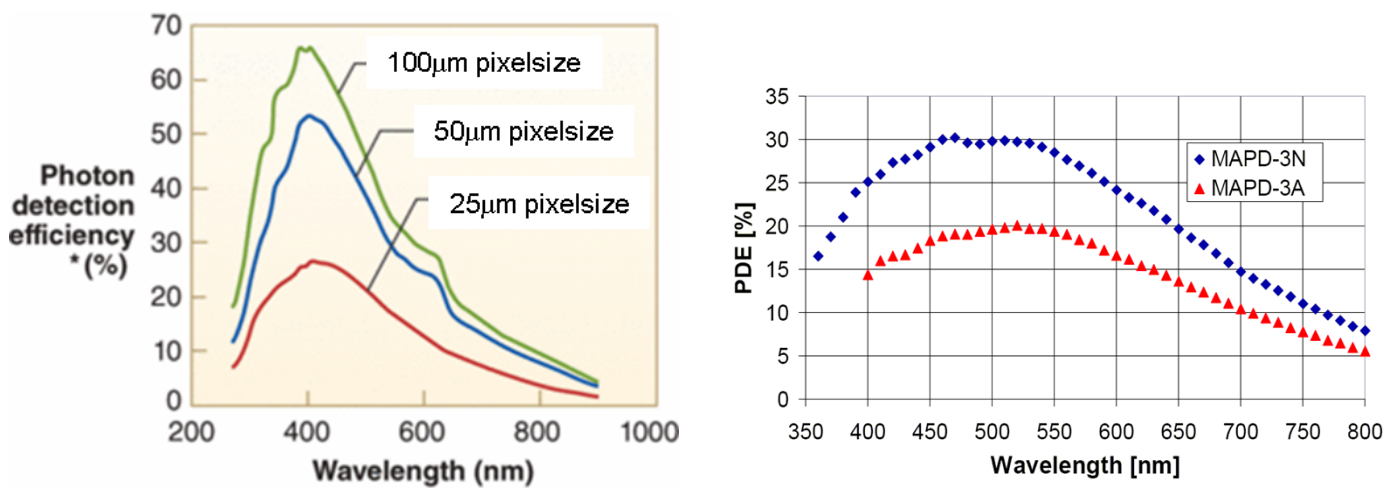

Figure 6: Left: Influence of the pixel size on the PDE of a SiPM. Right: Measured PDE values depending on the wavelength of the incident light measured for two different SiPM types (taken from [4]).

\subsection{Optical crosstalk}

As discussed in [5] $\approx 3 \cdot 10^{-5}$ photons with energies higher than $1.14 \mathrm{eV}$ are emitted per carrier crossing a p-n junction in silicon (Bremsstrahlung). Due to this estimate $10^{5}$ avalanche process produced carriers will create 3 photons of energies higher $1.14 \mathrm{eV}$, which are able to trigger 
an additional breakdown most likely in a neighbouring cell of the SiPM as schematically shown in Fig. 7. Due to the absorption length of light in silicon, internally produced photons with a wavelength in the region of $850 \mathrm{~nm}<\lambda<1100 \mathrm{~nm}$ most likely contribute to the internal optical crosstalk (details can be found in [6]): Photons with energies above $1.4 \mathrm{eV}$ have got an absorption length less than $10 \mu \mathrm{m}$ (absorption within the same pixel) and photons with energies below energies of $1.15 \mathrm{eV}$ have absorption lengths larger than $1 \mathrm{~mm}$ and are likely not to be absorbed in the device if its active area is smaller than the energy dependent absorption length. Therefore photons within this energy range could create satellite peaks even if only events triggered by one single carrier and therefore contribute to a pulse height spectrum as shown on the right side of Fig. 7 taken from [9].
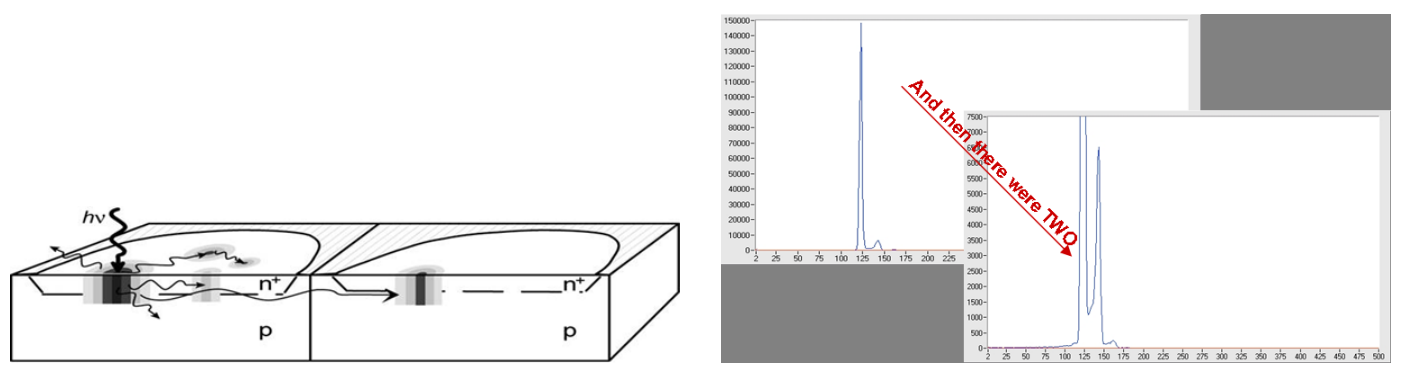

Figure 7: Left: Principle of the formation of optical crosstalk. Right: According to optical crosstalk events where a second and even a third pixel has fired are visible as satellite peaks (bottom). Pictures taken from [8], [9] respectively.

The process of optical crosstalk acts similar to an avalanche fluctuation in APDs and could therefore be understood as counterpart to the Excess Noise Factor mentioned in the section before. A reduction of optical crosstalk could be reached by separation of the individual pixels via trenches with the drawback of a decrease of the fill factor and, as a result of this, of the PDE of the device. Another simple possibility to reach less crosstalk is the reduction of the SiPM gain itself.

\subsection{Dark counts and afterpulses}

A breakdown in the device could be triggered by an incoming photon or any other generated free carrier e.g. by thermal excitation, leading to the problem, that the signal generated by a photon could not be distinguished from this 'noise' effect. This noise occurs randomly and its frequency is called dark count rate. Typical rate values are in the order of $100 \mathrm{kHz}$ up to several MHz per $\mathrm{mm}^{2}$ at room temperature. Another parameter which is of importance, not only if timing is the crucial issue of using a SiPM as readout device, is the occurance of afterpulses. The origin of this effect could be explained by the fact that a breakdown forms a plasma inside the Si volume (few thousand ${ }^{\circ} \mathrm{C}$ ). Caused by this plasma deep lying traps in the $\mathrm{Si}$ are filled by avalanching electrons, which are re-emitted after a certain time and create new avalanches, which are detected as afterpulses as shown in Fig. 8 taken from [13]. It could be easily seen, that the probability of afterpulsing increases with higher overvoltage (higher gain) applied to the device.

\subsection{Conclusion}

APDs as photodetection devices are suitabel for applications where high energy resolution is needed. The properties of these devices are well understood, and a usage of large quantities 


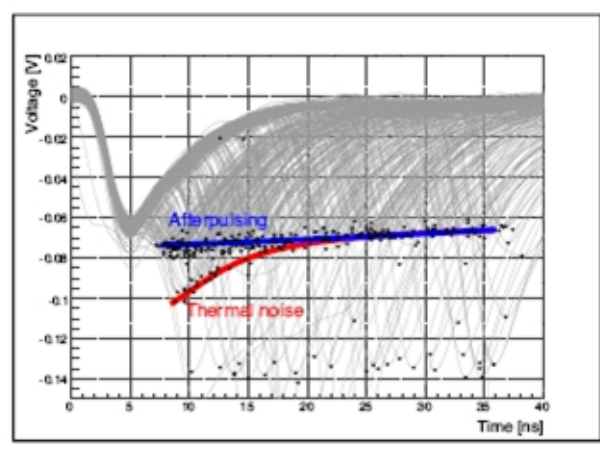

Figure 8: Several afterpulses (their amplitudes are emphasized in blue) occur during the measurement after the original signal of the incident 'single event' pulses were detected. Picture taken from [13], where also a detailed explanation of the used trigger procedure can be found.

in several experiments like CMS, PANDA and others is ongoing. The R\&D process of SiPMs is still going on with emphasis on the radiation hardness of these devices especially due to neutron irradiation and the reduction of crosstalk and afterpulsing. In the near future the achieved progress on this sector will make SiPMs usable in many applications, where time resolution and low light level detection will be the focal points of interest.

\section{References}

[1] S.L.Miller, Phys. Rev. 99 (4), 1955

[2] B. Dolgoshein et al, NIM A 504 (2003) pp. 48-52

[3] J.Marton, Stefan Meyer Institute for Subatomic Physics, Vienna

[4] Z.Sadygov, JINR - INR RAS - IP AZ - Zecotec Photonics Inc.

[5] A.Lacaita et al, IEEE Transaction on Electronic Devices, Vol.40, No.3 (1993)

[6] A.Nepomuk Otte, NIM A 610 (2009) pp. 105-109

[7] P.Barton et al, NIM A 610 (2009) pp. 393-396

[8] D.Renker, NIM A 567 (2006), pp. 48-56

[9] D.Renker, G-APD Workshop, GSI, 2009

[10] A.C.Guidice, M.Ghioni, S.Cova, Proceedings of ESSDERC03, Sept. 2003, pp.347-350

[11] Roland H.Haitz, Journal of Applied Physics, Vol.36, No.10, 1965

[12] Y. Kang et al. Appl, Phys. Lett., 83 (14) 2003

[13] H.Oide, Proceedings of PD07, 2007 